\title{
Rehabilitation: Keeping Pace with Societal Needs
}

\author{
JeMe Cioppa-Mosca, PT, MBA
}

Received: 5 August 2019/Accepted: 12 August 2019/Published online: 28 August 2019

(C) Hospital for Special Surgery 2019

The field of rehabilitation has grown substantially in the past century. Physical, occupational, and speech therapies all formed professional associations around the 1920s, with continued growth as World War I generated a large need for rehabilitative services $[1-3,7,8]$. Subsequent developments in medicine and governmental programs, the advent of World War II, and the outbreak of polio continued to advance the field of rehabilitation $[3,8]$. By the $1950 \mathrm{~s}$, rehabilitative services were understood to be of value, with practitioners keeping pace with the reconceptualization of disability, the increase in life expectancy, and the rising number of people living with chronic conditions $[3,6,8]$. Recognition that all people should pursue their greatest potential quality of life, no matter their age or physical barrier, has propelled the field of rehabilitation even further to successfully meet that mission.

Rehabilitation at Hospital for Special Surgery (HSS) first took root nearly 100 years ago, in 1924, as the Physiotherapy Department at the Hospital for the Ruptured and Crippled. It was identified in the hospital's 1924 annual report as necessary "to restore to proper function parts of the disabled human machine by the application of physical means." The physiotherapy department, initially a staff of 15 , contained four "divisions": massage and electrotherapy, phototherapy, hydrotherapy, and mechanotherapy. The occupational therapy department was established in the 1920s, and in the 1940s both departments were integrated into a combined Department of Physical Medicine. Also in the early 1940s, the hospital joined many others in offering "war emergency" training courses, to fulfill the great need for physical therapists in the armed forces [5].

Electronic supplementary material The online version of this article (https://doi.org/10.1007/s11420-019-09716-9) contains supplementary material, which is available to authorized users.

J. Cioppa-Mosca, PT, MBA $(\bowtie)$

Hospital For Special Surgery,

535 E. 70th St.,

New York, NY 10021, USA

e-mail: MoscaJ@HSS.EDU
Today, the HSS rehabilitation team includes over 375 occupational, physical, and speech therapists, performance and wellness practitioners, researchers, engineers, and administrative professionals in over a dozen offices of the HSS campus and partner sites across New York, New Jersey, and Connecticut.

The Department of Rehabilitation continues to be characterized by the fellowship and pride described by Kristian G. Hansson, MD, the first medical director for physiotherapy, in the 1930 HSS annual report:

Such progress is impossible without the full cooperation of the entire staff of the Department and it is a pleasure for me to testify to the splendid "esprit du corps" that prevails in the Department.

Recent innovations in rehabilitation at HSS include the use of motion-capture technology in investigating athletes' muscle activation during play, among other initiatives at the Leon Root, MD, Motion Analysis Laboratory; the use of a robotics rehabilitation system called Hunova to measure core strength and stability; the tracking of hand use with wearable technology for children with cerebral palsy; and the use of Targeted Muscle Re-innervation and Pattern Recognition Myoelectric software in collaboration with orthopedic surgeons to facilitate function in patients with osseointegration after amputation.

This special rehabilitation issue of HSS Journal ${ }^{\circledR}$ highlights the strides being made in rehabilitation both at HSS and beyond. Two articles illustrate advancing approaches to treatment and enhancing recovery of patients as they look beyond what has been accepted as standard: in the use of pose avoidance protocol following posterior approach total hip arthroplasty by Eannucci and colleagues (https://doi.org/ 10.1007/s11420-019-09708-9) and a structured program for women with osteoporosis by Sahni and Nieves (https:// doi.org/10.1007/s11420-019-09686-y). Articles by Mehta and colleagues (https://doi.org/10.1007/s11420-019-09702-1) and by Weinstock-Zlotnick and colleagues (https://doi.org/ $10.1007 / \mathrm{s} 11420-019-09705-\mathrm{y})$ explore the relationship 
between physical limitations and function in patients with osteoarthritis, for those with advanced osteoarthritis of the knee and varying stages of osteoarthritis of the first carpometacarpal joint in the hand, respectively. Successful recovery and discharge planning for arthroplasty patients is multifactorial [4, 9]; there is no consensus on the optimal timing and setting of rehabilitation after surgery [9], and the determinants of discharge destination after arthroplasty along with their concomitant outcomes and risks are a subject of much research [4]. Two studies in this issue explore those questions: Edwards and colleagues examine the clinical and social variables impacting discharge destination after bilateral arthroplasty (https://doi.org/10.1007/s11420-019-09701-2), and Pathak and colleagues consider the relationship between timing of post-discharge rehabilitation and unplanned readmissions (https://doi.org/10.1007/s11420-019-09685-z). Both provide important considerations in planning successful outcomes.

Two studies explore a game-changing innovation in rehabilitative care for arthroplasty patients: telehealth. Advances in technology and the need to provide value-based care have resulted in the remote delivery of care. The ability to use computers, phones, or tablets to effectively supervise post-operative recovery and minimize complications and readmissions is both valuable and timely. Two studies in this issue offer a glimpse into the implications and growing potential of telehealth care delivery after hip or knee arthroplasty. Kuether and colleagues present the results of a pilot program in telerehabilitation after primary total hip and knee arthroplasty using a remotely managed in-home technology system (https://doi.org/10.1007/s11420-01909715-w). Fisher and colleagues present their findings of the recent launch of a telehealth program called HSS@Home, which uses the patient's existing technology for remote care after arthroplasty (https://doi.org/10.1007/ s11420-019-09714-x). Both studies demonstrated favorable outcomes, highlighting the potentially vast implications for the applicability of telerehabilitation in orthopedics.

Rehabilitation's trajectory for continued evolution is exciting. Growth keeps pace with societal needs, opportunities for interdisciplinary collaboration, technology, and research [10]. The goal of helping people to perform at their desired functional capacity has become the endeavor of health care professionals worldwide [10]. Rehabilitation is proud to play a pivotal role in making that happen.

Thank you to all my rehabilitation colleagues who have contributed to this special issue of HSS Journal.

\section{Compliance with Ethical Standards}

Conflict of Interest: JeMe Cioppa-Mosca, PT, MBA, declares that she has no conflicts of interest.

\section{Human/Animal Rights: N/A}

Informed Consent: N/A

Required Author Forms Disclosure forms provided by the authors are available with the online version of this article.

\section{References}

1. American Physical Therapy Association. APTA history. Available at https://www.apta.org/History. Accessed July 21, 2019.

2. American Speech-Language-Hearing Association. History of ASHA. Available at https://www.asha.org/about/history. Accessed July 21, 2019.

3. Cole TM, Kewman D, and Boninger ML. Development of medical rehabilitation research in 20th-century America. Am J Phys Med Rehabil. 2005;84(12): 940-954.

4. Keswani A, Tasi MC, Fields A, Lovy AJ, Moucha CS, Bozic KJ. Discharge destination after total joint arthroplasty: an analysis of postdischarge outcomes, placement risk factors, and recent trends. J Arthroplasty. 2016;31(6):11551162.

5. Levine DB. The Hospital for the Ruptured and Crippled renamed the Hospital for Special Surgery 1940; the war years 1941-1945. HSS J. 2009;5(1):1-8.

6. Stucki G, Bickenbach J, Gutenbrunner C, Melvin J. Rehabilitation: the health strategy of the 21 st century. J Rehab Med. 2018;50(4):309-316.

7. The American Occupational Therapy Association, Inc. History of AOTA Accreditation. The American Occupational Therapy Association. https://www.aota.org/Education-Careers/Accreditation/ Overview/History.aspx. Accessed 7/21/19.

8. Verville R. War, politics, and philanthropy: the history of rehabilitation medicine. Lanham: University Press of America; 2009.

9. Westby MD, Brittain A, Backman CL. Expert consensus on best practices for post-acute rehabilitation after total hip and knee arthroplasty: a Canada and United States delphi study. Arthritis Care Res (Hoboken). 2014;66(3):411-423.

10. World Health Organization. International classification of functioning, disability and health: ICF. Geneva: World Health Organization, 2001. Available at https://www.who.int/classifications/icf/en. Accessed July 21, 2019. 